\title{
Severe myocarditis of possible human herpesviruses 6 and 7 etiology in an 8-year-old immunocompetent child
}

\author{
Radosław Jaworski ${ }^{1}$, Katarzyna Pręgowska², Beata Kasztelewicz³, \\ Monika Kowalczyk-Domagała², Andrzej Kansy'1, Grażyna Brzezińska-Rajszys², \\ Katarzyna Dzierżanowska-Fangrat ${ }^{3}$ \\ 'Department of Cardiothoracic Surgery, Children's Memorial Health Institute, Warsaw, Poland \\ 2Department of Cardiology, Children's Memorial Health Institute, Warsaw, Poland \\ ${ }^{3}$ Department of Clinical Microbiology and Immunology, Children's Memorial Health Institute, Warsaw, Poland
}

\section{ABSTRACT}

In this paper we present a case of an 8-year-old immunocompetent boy with acute myocarditis caused probably by concomitant human herpesvirus 6 (HHV-6) with human herpesvirus 7 (HHV-7) infection. The clinical manifestation included flu-like symptoms and fulminant heart failure, which required admission to an intensive care setting. Aggressive supportive heart failure management with administration of inotropes and antiviral and antibacterial treatment were applied. After implementation of therapy, the gradual improvement of heart function and patient's condition was observed and the patient did not require Extracorporeal Membrane Oxygenation (ECMO) support. Two months after the symptoms' onset, the patient was discharged home in a good general condition.

KEY WORDS:

HHV-6, HHV-7, children, myocarditis, congestive heart failure.

\section{INTRODUCTION}

Recently, both human herpesviruses 6 (HHV-6) and human herpesviruses 7 (HHV-7) have been reported as a possible cause of myocarditis. However, little is known about acute myocarditis in children concomitantly infected with HHV-6 and HHV-7. In this paper we present a case of an immunocompetent 9 -year-old boy with acute myocarditis of nearly fatal course and concomitant HHV-6 and HHV-7 infection.

\section{CASE REPORT}

An eight-year old febrile boy with a 3 week-history of flu-like clinical symptoms and 3 day-history of chest pain and vomiting was admitted to our hospital. On admission, the child was fretful and weakened, presenting with gallop- ing heart rhythm 120 beats per minute, intensified vesicular breathing, hepatomegaly (liver edge palpated $3 \mathrm{~cm}$ below the right costal margin at the midclavicular line) and colder distal parts of limbs. Chest pain was not connected with body position and escalating during deep breathing. Child's oral examination showed severe dental caries. Blood tests showed elevated inflammatory markers (C-reactive protein $250 \mathrm{mg} / \mathrm{l}$ and procalcitonin $1.07 \mathrm{ng} / \mathrm{ml}$ ), and highly elevated troponin T (12407 pg/ml), CK-MB (19.6 ng/ml), NTproBNP $(11049 \mathrm{pg} / \mathrm{ml})$ and LDH $(453 \mathrm{U} / \mathrm{I})$. On chest X-ray, parenchymal opacity with cardiothoracic ratio 0,61 and pleural effusion in right pleural space were present. On electrocardiogram, abnormal repolarization period was detected, with inverted $\mathrm{T}$ waves from $\mathrm{V} 1$ to $\mathrm{V} 5$ and flat $\mathrm{T}$ wave in V6. Echocardiography revealed impaired left ventricle systolic function, with shortening fraction and ejection fraction 12 and 36\%, respectively (Figure 1).

\section{ADDRESS FOR CORRESPONDENCE:}

Katarzyna Pręgowska, Department of Cardiology, Children's Memorial Health Institute, Warsaw, Poland, e-mail: k.pregowska@ipczd.pl 


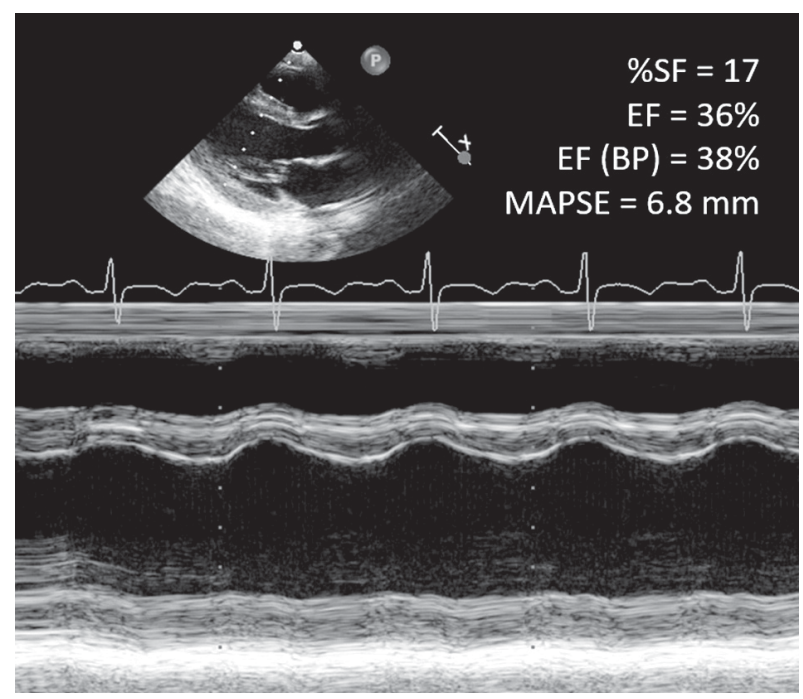

FIGURE 1. Left ventricle function assessed by m-mode presentation at the time of admission (\%SF - \% of shortening fraction; EF - ejection fraction; EF (BP) - ejection fraction [Biplane Simpson method]; MAPSE - mitral annular plane systolic excursion)

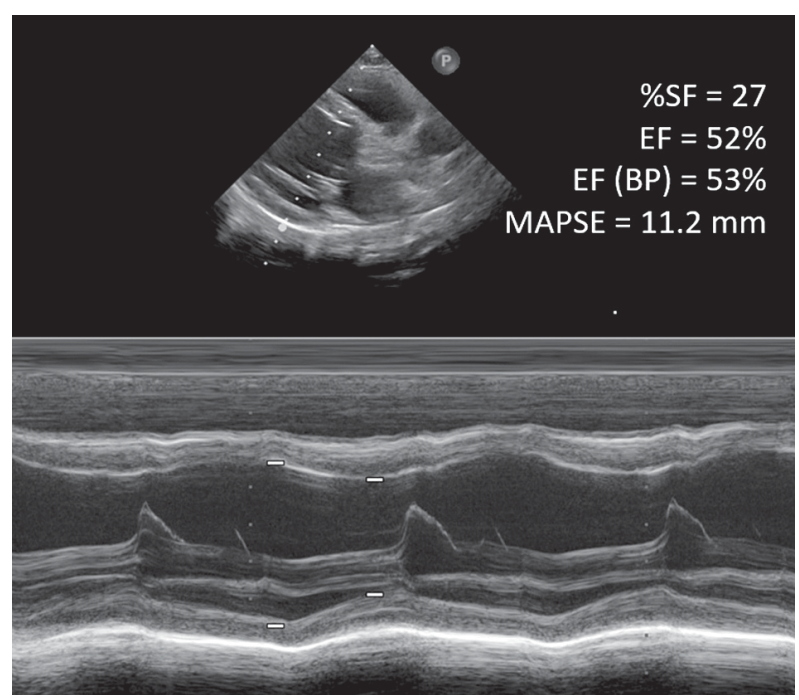

FIGURE 2. Left ventricle function assessed by m-mode presentation at the time of improvement (\%SF - \% of shortening fraction; $\mathrm{EF}-$ ejection fraction; EF (BP) - ejection fraction [Biplane Simpson method]; MAPSE - mitral annular plane systolic excursion)

Intravenous infusion of milrinon $0,4 \mu \mathrm{g} / \mathrm{kg} / \mathrm{min}$ and catecholamines afterwards (adrenaline $0,25 \mu \mathrm{g} / \mathrm{kg} / \mathrm{min}$, noradrenaline $0,32 \mu \mathrm{g} / \mathrm{kg} / \mathrm{min}$, dopamine $5 \mu \mathrm{g} / \mathrm{kg} / \mathrm{min}$ ) were started.

Blood was drawn for microbiological analysis, and an empirical treatment with ceftriaxone and vancomycin was started. On the second day, patient's status deteriorated rapidly and he required an intensive care including a mechanical ventilation. Antibiotics were changed to linezolid and levofloxacin and intravenous infusion of immunoglobulins $2 \mathrm{~g} / \mathrm{kg}$ was given. Due to acute renal failure, renal replacement therapy (continuous veno-venous hemodiafiltration, CVVHDF) was also implemented and continued for 3 days.

All blood cultures taken on admission were negative (one probe was positive for Staphylococcus epidermidis

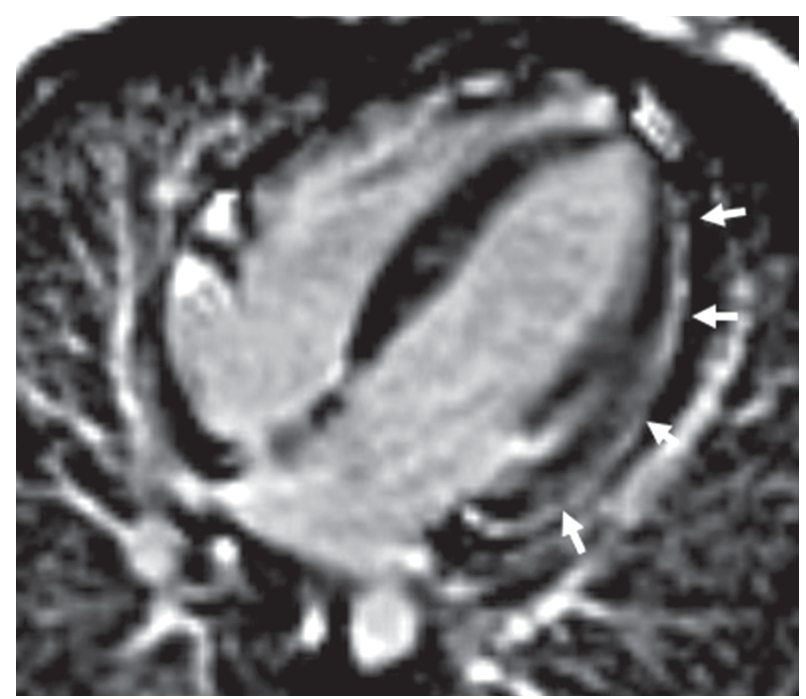

FIGURE 3. Cardiac magnetic resonance performed 8 weeks after acute onset of myocarditis, showing typical signs of healed myocarditis: arrows point myocardial scar within the infero-lateral wall

but was described as sample contamination). Multiplex real-time PCR in whole blood revealed coinfection with HHV-6 and HHV-7, while results for adenovirus, enterovirus, parvovirus B19, cytomegalovirus and Epstein-Barr virus were negative. Quantitative real time PCR in whole blood revealed viral load of $<320$ copies $/ \mathrm{ml}$ and 365 copies/ml for HHV-6 and HHV-7, respectively. Once the diagnosis was established acyclovir was started and continued for 14 days.

Due to severe patient's condition, the arteriovenous Extracorporeal Membrane Oxygenation (ECMO) was considered, but finally not applied. Due to the same reason, the heart biopsy was not performed.

After implementation of the antiviral and antibacterial therapy, patient's general status started improving slowly and so did laboratory and echo indices. The duration of mechanical ventilation was 10 days. After 6 weeks, shortening fraction and ejection fraction on echocardiography were 27 and 52\%, respectively (Figure 2). Cardiac magnetic resonance (CMR) performed 8 weeks after the heart failure onset showed typical signs of healed myocarditis (Figure 3 ). Repeated quantification of $\mathrm{HHV}-6 / 7$ viremia in whole blood performed within 2 months revealed stable viral loads of $<320$ copies/mL for HHV-6 and 933 copies/ml (less than $0.5 \log$ increase) for HHV-7. Both viral loads were undetectable in plasma sample tested in parallel. After 10 weeks, the boy was discharged home in a good general condition and remains in the close clinical follow-up.

\section{DISCUSSION}

The most common infectious agents of pediatric myocarditis are enteroviruses, parvovirus B19, adenoviruses, cytomegalovirus and HHV-6. It is estimated that among patients with viral myocarditis one-third of them experience complete recovery, one-third has residual cardiac 
dysfunction and one-third shows cardiac failure resulting in death or heart transplantation [1].

There are few reports on survivors of severe myocarditis complicated by acute heart failure caused by HHV-6 and HHV-7, especially in pediatric population.

There are two identified subtypes of HHV-6: HHV-6A and HHV-6B. HHV-6A may cause direct infection of cardiomyocytes whereas the other infects the vascular endothelium and causes endothelial and diastolic dysfunction [2]. Recently, HHV-7 has been also reported as a possible cause of this disease [3]. Given the immunomodulatory properties of both HHV-6 and HHV-7, it has been suggested that not only active but also persistent infection may have putative indirect pathogenic effect in myocarditis and cardiomyopathy $[4,5]$. Relatively low HHV-6/7 viremia observed in our patient may reflect either active infection well controlled by immune response or latent infection. As there is no established whole blood viral load threshold to distinguish between active and latent infection, repeated viral load testing on consecutive whole blood specimens or viral load testing in plasma sample may be informative. Stable (or below lower limit of quantification) HHV-6/7 viral load in whole blood or undetectable viral load in plasma imply latent infection. Nevertheless, negative findings in the plasma or low viral load in whole blood do not rule out a localized active infection in an organ (e.g. myocardium). It is suggested that the value of viremia does not need to be significantly high to establish the diagnosis of active infection [4].

HHV-6 infection treatment consists classically of the antivirals such ganciclovir, cidofovir, and foscarnet, however the therapy is often limited by toxicities [6]. With probable HHV-6 infection and considered toxicity of other antivirals, acyclovir was prescribed in our patient. This antiviral drug is active against HHV-6 in vitro, but only at very high concentrations that can hardly be obtained in body fluids in vivo. Therefore, acyclovir is usually not considered to treat HHV-6 infections. In our opinion, the use of acyclovir in this certain case could finally highly contribute to clinical improvement, as well as IVIG and wide-spectrum antibiotics intake.

Recently an interesting case in which the antimalarial drug artesunate was used to treat HHV-6 viremia was reported [7].

Treatment strategies in severe cases of heart failure due to acute myocarditis comprise mechanical support or even heart transplantation [8]. A successful implantation of left ventricular assist device (LVAD) was described as a bridge-to-recovery [9]. In the case of our patient, in the acute stage of the disease he was qualified for ECMO implantation, but continuous medical observation allowed detection of slight but consistent improvement. Chosen strategy of "watchful waiting" let us resign from this procedure.

Although acute systolic heart failure precludes a full diagnostic workup, including endomyocardial biopsy
(EMB), we decided not to perform this highly invasive procedure in our patient. The boy faced a rapidly deteriorating condition and biopsy in acute infection phase would be of a significant risk. Although still histopathological testing remains the gold standard for the diagnosis of myocarditis, EMB is not performed in most myocarditis cases involving children in the United States [10]. In our patient, the diagnosis of myocarditis was finally confirmed by CMR, showing myocardial scar within the infero-lateral wall (Figure 3). In myocarditis, CMR is an important diagnostic tool complementary to echocardiography; it contributes to improvement of the diagnostic accuracy, allowing tissue characterization and giving the opportunity to evaluate functional consequences of the inflammatory process.

This paper may add substantial knowledge to the clinical course, treatment and outcome of acute myocarditis of nearly fatal course of possible concomitant HHV-6 and HHV-7 etiology. To our knowledge, there is a lack of such data in pediatric population.

\section{ACKNOWLEDGMENTS}

Cardiac MR image by courtesy of Joanna Petryka-Mazurkiewicz, MD, PhD.

\section{REFERENCES}

1. Wheeler DS, Kooy NW. A formidable challenge: The diagnosis and treatment of viral myocarditis in children. Crit Care Clin 2003; 19: 365-391.

2. Tschöpe C, Bock CT, Kasner M, et al. High prevalence of cardiac parvovirus B19 infection in patients with isolated left ventricular diastolic dysfunction. Circulation 2005; 111: 879-886.

3. Ozdemir R, Kucuk M, Dibeklioglu SE. Report of a Myocarditis Outbreak among Pediatric Patients: Human Herpesvirus 7 as a Causative Agent? J Trop Pediatr 2018; 64: 468-471.

4. Agut H, Bonnafous P, Gautheret-Dejean A. Laboratory and clinical aspects of human herpesvirus 6 infections. Clin Microbiol Rev 2015; 28: 313-335.

5. Lassner D, Krueger GRF, Maximilian Buja L, et al. HHV-6 and HHV-7 in Cardiovascular Diseases and Cardiomyopathies. In: $\mathrm{Hu}-$ man Herpesviruses HHV-6A, HHV-6B, and HHV-7, Third Edition. Elsevier B.V. 2014; 267-280.

6. Reddy S, Eliassen E, Krueger GR, et al. Human herpesvirus 6-induced inflammatory cardiomyopathy in immunocompetent children. Ann Pediatr Cardiol 2017; 10: 259-268.

7. Hakacova N, Klingel K, Kandolf R, et al. First therapeutic use of Artesunate in treatment of human herpesvirus 6B myocarditis in a child. J Clin Virol 2013; 57: 157-160.

8. Kindermann I, Barth C, Mahfoud F, et al. Update on myocarditis. J Am Coll Cardiol 2012; 59: 779-792.

9. Cavigelli-Brunner A, Schweiger M, Knirsch W, et al. VAD as bridge to recovery in anthracycline-induced cardiomyopathy and HHV6 myocarditis. Pediatrics 2014; 134: e894-e899.

10. Das BB. Role of endomyocardial biopsy for children presenting with acute systolic heart failure. Pediatr Cardiol 2014; 35: 191-196. 